Entropy 2004, 6, 96-101

Entropy

ISSN 1099-4300

www.mdpi.org/entropy/

Full Paper

\title{
Smoluchowski's Trapdoor
}

\section{Lyndsay G M Gordon}

11 Guildford Tce., Thorndon, Wellington, New Zealand. E-mail: lgordon@actrix.gen.nz

Received: 5 September 2003 / Accepted: 25 December 2003/ Published: 22 March 2004

\begin{abstract}
A mechanism of a gated pore in a membrane is described. Fluxes of gas molecules pass through the pore and produce a pressure gradient in a process that challenges the second law.
\end{abstract}

Keywords: second law, Smoluchowski, Maxwell, nanotechnology

\section{Introduction}

Skordos and Zurek [1], in their deliberations on Smoluchowski's trapdoor, fall into the same syllogistic error as does Feynman [2,3] in his discussions of a ratchet and pawl within the microdomain. Both sets of workers incorrectly perceive their illustrations as universal representaions of phenomena they wish to explain, otherwise their models would be no importance. In an accompanying paper within this special issue [4] and elsewhere [5], Feynman's contribution to the development of our understanding of fluctuations and the limitations of his ratchet and pawl are discussed. It is doubtful whether the model of Skordos and Zurek can be developed further to achieve a similar goal since it is neither realistic nor does it have as a basis the Maxwellian idea that molecules should be handled one at a time. Their device is a mini-system where transported molecules are treated en masse. Certainly, the motion of the trapdoor is Brownian, but the project misses the point that a Maxwellian 
valve [6] requires gas molecules to be handled individually. An earlier model of a Smoluchowski trapdoor [7] which, set in the pore of a membrane, avoided this problem by allowing the opening process to be generated from collisions with individual molecules.

It is not the author's wish here to proceed through the quantitative treatment of that early work [7], but to give a greater credence to the mechanism described there by linking it to a set of wellformulated organic reactions.

\section{Smoluchowski's Trapdoor revisited}

For aqueous systems, the formation of pores by polypeptides in bilayer lipid membranes has been well-documented [8]. For example, gramicidin can form pores in membranes of glycerol monooleate where the membrane thickness is approximately $3 \mathrm{~nm}$. The diameter of the pore is such that only small cations can pass through. In nerve cells, separate pores exist for the $\mathrm{Na}$ and $\mathrm{K}$ ions to traverse the cell membrane and are known to pass in single file. The previously reported trapdoor model [7] was composed with this background in mind, but was adapted for a gaseous system and a developing nanotechnology.

In brief, the system (Fig. 1) is composed of two gaseous compartments, A and B, separated by a heat conducting membrane containing pores for the transport of gas molecules. The heat conduction of the membrane ensures a single heat source. Within each pore is a trapdoor composed of one or more atoms. Except for the asymmetry of the movement of the trapdoor (see Fig. 1), simple physicochemical properties pertain and are expressed in under-lined text.

(i) The trapdoor can be opened from the impact of a gas molecule. That is, the translational energy of the gas molecule can be transformed into the rotational energy of the trapdoor.

(ii) The impacts of molecules from compartments A and B have equal probabilities of opening the trapdoor but not for closing it as described below.

The passage of a gas molecule through the membrane is essentially a chemical reaction due to a change of configuration of the system and an accompanying change in potential energy, and we can write for the forward and reverse reactions : 


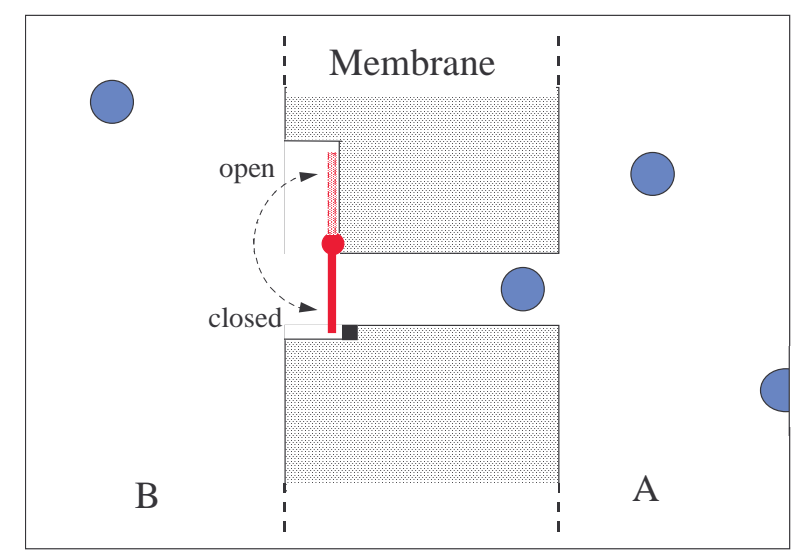

FIGURE 1. A magnified view of the membrane pore and the gating particle or trapdoor shown in the closed position.. The membrane contains a large number of gated pores oriented in the same direction. The resident times in open and closed positions are long in comparison with transition times.

$$
\mathrm{G}_{\mathrm{A}} \leftrightarrows \mathrm{G}_{\mathrm{B}}
$$

where $\mathrm{G}$ is the gas and the subscripts denote location. This reaction has two sets of rate constants: one set for when the pore is open and the other for when the pore is closed.

The second law of thermodynamics imposes the following condition.

(iii) During the open state of the trapdoor, the rate constants for gas transfer are equal.

For us to show that this particular Smoluchowski trapdoor is a Maxwellian valve, we are required to prove an inequality between the second pair of rate constants where collisions with the trapdoor are involved.

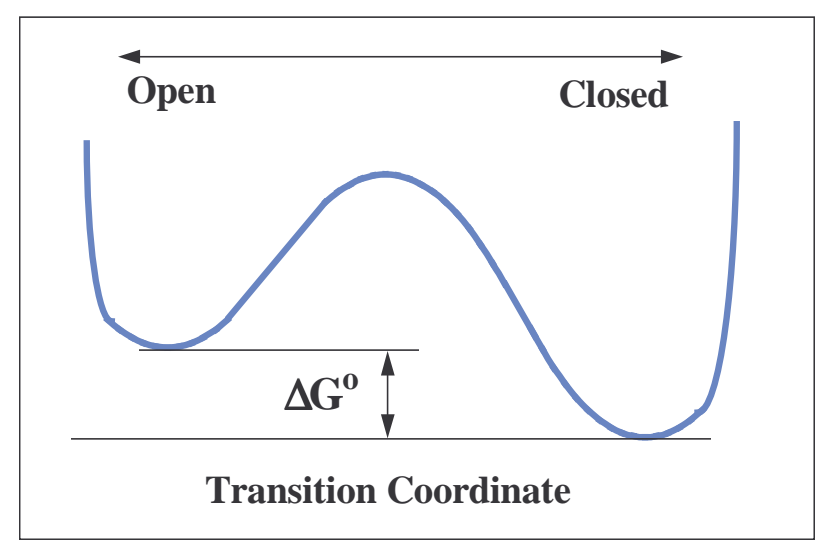

FIGURE 2. The potential energy profile of the trapdoor between its open and closed states. At the extremes of the profile the potential energy rises steeply above the activation energy barrier. The standard free energy (potential energy) between states is shown. 
The trapdoor in the pore does not swing to and fro like a macroscopic trapdoor, which continues to oscillate until frictional drag brings it to a halt. Here the molecular trapdoor moves between its two states within the restrictions imposed by quantum mechanics (Fig. 2).

The state of the trapdoor is governed by the absorption and desorption of energy by conduction (through connection with the membrane), by radiation (within the system as a whole) and by convection (via collisions with the gas molecules). The closed and open states of the trapdoor are in equilibrium and the transit of gas molecules per se does not alter this condition.

\section{Steriochemistry}

The two types of the transitions of gas molecules through the pore are identical in property with other steriochemical reactions. That is, where interactions are dependent on the orientation(s) of reactant(s) along the approach path. Because many readers will not be familiar with this field of science, examples of steriochemical organic reactions shall be explained and compared with the passage of gas molecules through the pore.

Attention to $S_{N} 1$ and $S_{N} 2$ reactions of optical isomers provides an excellent basis for discussion [9]. Firstly, when R-2-bromooctane (with its central carbon atom surrounded tetrahedrally by $\mathrm{H}, \mathrm{CH}_{3}$, $\mathrm{Br}$ and $\mathrm{C}_{6} \mathrm{H}_{13}$ ) reacts with hydroxyl ions it forms only S-2-octanol in an $\mathrm{S}_{\mathrm{N}} 2$ reaction. That is, $\mathrm{OH}$ replaces $\mathrm{Br}$ on the central carbon atom and at the same time the configuration is changed from $\mathrm{R}$ (rectus) to $\mathrm{S}$ (sinister). Here the optical rotation changes from negative to positive.

An example of an $\mathrm{S}_{\mathrm{N}} 1$ reaction is that of S-3-bromo-3-methyl hexane (central carbon atom surrounded by $\mathrm{Br}, \mathrm{CH}_{3}, \mathrm{C}_{2} \mathrm{H}_{5}$, and $\mathrm{C}_{3} \mathrm{H}_{7}$ ) with water in acetone as solvent, where the $\mathrm{Br}$ is replaced by $\mathrm{OH}$ to form equal amounts of R- and S-methyl hexanol. Racemization, indicated by a reduction of optical activity to zero, occurs in this study but not in the former. Why?

The mechanisms for these two reactions $\left(S_{N} 1\right.$ and $\left.S_{N} 2\right)$ both pass through similar activated complexes where the 3 permanent groups on the central carbon atom and the carbon atom itself form a planar group. That is, bond directions move away from their tetrahedral orientations to lie in the one plane. In the first reaction the leaving group $(\mathrm{Br})$ does not dissociate until the new group $(\mathrm{OH})$ has formed a linkage with the central carbon atom. Therefore, to react, the $(\mathrm{OH})$ group must approach the central carbon atom from the opposite side of the plane to the $(\mathrm{Br})$ group. In the second reaction the $(\mathrm{Br}$ 
-C) bond breaks before the $(\mathrm{OH})$ has had time to react and the planar group is left isolated until the reaction occurs. As the $(\mathrm{Br})$ is not attached, an $(\mathrm{OH})$ can approach from either side of the plane to form an R- or S- type tetrahedral molecule and thus both forms are produced in equal quantities.

When the trapdoor is open, reactions can occur with gas molecules passing through from either compartment without obstruction like in the $\mathrm{S}_{\mathrm{N}} 1$ reaction. The process is analogous to racemization.

In the situation where the trapdoor is closed, a gas molecule can strike from either side. For the closed trapdoor to open after a collision with a gas molecule from compartment $\mathrm{B}$, the trapdoor must move initially further to the right (Fig. 2) so that it exceeds the height of the activation energy barrier on the extreme right of the reaction coordinate. This gain in energy allows the trapdoor to rebound over the activation energy barrier. The return movement of the trapdoor carries the gas molecule with it, if it has not already rebounded into compartment B from the time of impact. This particular gas molecule simply does not have the correct velocity (incorrect direction) to pass through the pore after it has opened the trapdoor. Furthermore the trapdoor can not open without sweeping through the space between the trapdoor and compartment $B$. Thus, analogous to the $S_{N} 2$ reaction, the gas molecule has to approach with the correct orientation and the correct velocity for it to collide with the trapdoor and simultaneously pass through the pore. This can happen only when the approach of the gas molecule is from compartment A.

A quantitative treatment [7] shows that the lower the potential energy of the trapdoor in the closed position with respect to the open position, the greater the asymmetry produced by the trapdoor in a process contrary to the second law. The potential energies of the trapdoor are directly related to the resident times in the two positions.

Not included in the above description is the interaction of the open trapdoor with gas molecules. Only molecules from B are involved (Fig. 2) and they are capable of closing the trapdoor in a manner similar to their contribution to the opening process. These gas molecules are also returned to compartment B.

In summary, the trapdoor model appears to succeed because packets of favourable energy arrive at the trapdoor at the same time as the transported masses since the masses carry the energy. The mass to be transferred and the energy to open the trapdoor need to be firmly correlated in the elastic collisions. However, the efficiency of the process is decreased by packets of energy being exchanged via conduction and radiation between the trapdoor and respectively the membrane and the system as a 
whole. Both of these methods are not correlated with the movements of gas molecules and therefore, they tend to increase the proportion of $\mathrm{S}_{\mathrm{N}} 1$ type reactions. Like Feynman, Smoluchowski [10] concluded that any device that would use fluctuations to convert heat completely into work on a macroscopic scale, would be subject to the same chance fluctuations and fail. In this paper and in others, the author has attempted to remove or reduce the element of chance and to substitute the relevant correlation.

\section{References}

1. Skordos, P. A.; Zurek, W. H. Maxwell's demon, rectifiers and the second law: Computer simulation of Smoluchowski's trapdoor. Am. J. Phys. 1992, 60, 876-882.

2. Feynman, R. P. The character of physical law; M.I.T. Press, Cambridge, MA, 1967.

3. Feynman, R.P.; Leighton, R. B.; Sands, M. The Feynman Lectures on Physics; Addison-Wesley: Reading, Mass. , 1963; Vol. 1, Chapter 46, pp 1-9.

4. Gordon, L. G. M. The decrease in entropy via fluctuations. Entropy 2004, this issue

5. Gordon, L.G.M. Brownian Motion and Microscopic Irreversibility. Found. Phys. 1981, 11, 103-113.

6. Maxwell, J. C. Theory of Heat; Longmans, Green and Co: London, 1872; 308-309.

Maxwell, J. C. In Life and Scientific Work of Peter Guthrie Tait. Knott, C. G.; Cambridge Univ. Press, London, 1911; Letter to P. G. Tait.

7. Gordon, L.G.M. Maxwell's demon and Detailed balancing. Found. Phys. 1983, 13, 989-997.

8. Hladky, S.B.; Gordon, L. G. M.; Haydon, D. A. Molecular mechanisms of ion transport in lipid membranes. Ann. Rev. Phys. Chem. 1974, 25, 11-38.

9. Solomons, T. W. G. Fundamentals of Organic Chemistry; John Wiley \& Sons: New York, 1982, 256-7

10. Smoluchowski, M. v. Experimentell nachweisbare, der üblichen Thermodynamik widersprechende Molekular-phänomene 1912, 13, 1069-1080.

(C) 2004 by MDPI (http://www.mdpi.org). Reproduction for noncommercial purposes permitted. 